Revista de Matemática: Teoría y Aplicaciones 1996 3(1) : 11-20

CIMPA - UCR ISSN: 1409-2433

\title{
ON SPACE-TIME PROPERTIES OF SOLUTIONS FOR NONLINEAR EVOLUTIONARY EQUATIONS WITH RANDOM INITIAL DATA*
}

\author{
KYRILL L. VANINSKY ${ }^{\dagger}$
}

\begin{abstract}
We consider space-time properties of periodic solutions of nonlinear wave equations, nonlinear Schrödinger equations and KdV-type equations with initial data from the support of the Gibbs' measure. For the wave and Schrödinger equations we establish the best Hölder exponents. We also discuss KdV-type equations which are more difficult due to a presence of the derivative in the nonlinearity.
\end{abstract}

Keywords: space-time properties; Hölder exponents; nonlinear evolutionary equations.

\section{Resumen}

Consideramos las propiedades en espacio tiempo de las soluciones periódicas de ecuaciones de onda no lineales, ecuaciones no lineales de Schrödinger y ecuaciones de tipo KdV con datos iniciales del soporte de la medida de Gibbs. Para las ecuaciones de onda y de Schrödinger establecemos los mejores exponentes de Hölder. También discutimos las ecuaciones de tipo $\mathrm{KdV}$, que son más difíciles debido a la presencia de la derivada en la no linealidad.

Palabras clave: propiedades espacio-tiempo; exponentes de Hölder; ecuaciones no lineales evolutivas.

Mathematics Subject Classification: 35B65, 82C05.

\footnotetext{
${ }^{*}$ The author would like to thank IHES where the paper was written for hospitality. The work is supported by NSF grant DMS-9501002.

${ }^{\dagger}$ Kansas State University, Manhattan KS 66506, United States of America; vaninsky@math.ksu.edu
} 


\section{Nonlinear wave equations}

Consider the ID nonlinear wave equation

$$
Q_{t t}-Q_{x x}+f(Q)=0
$$

with periodic boundary conditions $Q(0, t)=Q(2 \pi, t)$. The equation can be written in the Hamiltonian form

$$
\begin{aligned}
Q_{t} & =\{Q, H\}, \\
P_{t} & =\{P, H\},
\end{aligned}
$$

with $H(Q, P)=\int_{0}^{2 \pi}\left[\frac{P^{2}}{2}+\frac{Q^{2}}{2}+F(Q)\right], F^{\prime}=f$, and a classical bracket

$$
\{A, B\}=\int_{0}^{2 \pi}\left[\frac{\partial A}{\partial Q(x)} \frac{\partial B}{\partial P(x)}-\frac{\partial A}{\partial P(x)} \frac{\partial B}{\partial Q(x)}\right] d x .
$$

An invariant Gibbs' state $e^{-H} d^{\infty} Q d^{\infty} P$ in the space ${ }^{1}$ of pairs $(Q, P) \in H^{0} \times H^{-1}$ was constructed in [6] under the assumption that $f(Q)$ is an odd locally Lipschitz function such that $f(Q) \geq k Q$, for some $k>0$ and big $Q$. To simplify the proof we impose an additional condition on the growth of $f$ at infinity: $f(Q) \leq C(\epsilon) e^{\epsilon Q^{2}}$, for any $\epsilon>0$. The Gibbs' state is a product measure: the $Q$ component is $e^{-\int Q_{x}^{2} / 2} d^{\infty} Q$, a circular Brownian motion with uniformly distributed initial position multiplied by the Radon-Nikodym factor $e^{-\int F(Q)}$ and "white noise" measure $e^{-\int P^{2} / 2} d^{\infty} P$ on the $P$ component.

Using variation of parameters we can write the original differential equation in the integral form

$$
\begin{aligned}
Q(x, t) & =\frac{\sin \sqrt{-\partial_{x}^{2}} t}{\sqrt{-\partial_{x}^{2}}} P_{0}(x)+\cos \sqrt{-\partial_{x}^{2}} t Q_{0}(x)-\int_{0}^{t} d s \frac{1}{2} \int_{x-(t-s)}^{x+(t-s)} d y f(Q(y, s)) \\
& =Q_{W}(x, t)+N(x, t)
\end{aligned}
$$

where $\left(Q_{0}, P_{0}\right)$ are the initial data. The term $Q_{W}(x, t)$ corresponding to the linear wave equation satisfies the Hölder condition

$$
\left|Q_{W}\left(x_{1}, t_{1}\right)-Q_{W}\left(x_{2}, t_{2}\right)\right| \leq K\left(\left|x_{1}-x_{2}\right|^{\beta_{1}}+\left|t_{1}-t_{2}\right|^{\beta_{2}}\right),
$$

with $0<\beta_{1}, \beta_{2}<\frac{1}{2}$ and some random constant $K, E K^{2}<\infty$, which depends on the $\beta$ 's. The bound $\frac{1}{2}$ is optimal in a sense that we can not have (1) with some $\beta_{1}>\frac{1}{2}$, or $\beta_{2}>\frac{1}{2}$ and random $K, E K^{2}<\infty$. The nonlinear part $N(x, t)$ is a differentiable function of $x$ and $t$. The derivatives $\partial_{x} N(x, t)$ and $\partial_{t} N(x, t)$ satisfy (1) with the same exponents not exceeding $\frac{1}{2}$. These simply means that the local structure of the field $Q(x, t)$ is completely determined by the term $Q_{W}(x, t)$ corresponding to the linear wave equation. We split the proof of these facts in three different steps.

\footnotetext{
${ }^{1} H^{s}$ is a standard Sobolev's space, i.e. $Q(x) \in H^{s}$ if $\left(1-\Delta^{2}\right)^{s / 2} Q(x) \in L^{2}[0,2 \pi]$.
} 


\section{Step 1}

In the proof of the statement concerning (1) we use Kolmogoroff's criteria of continuity, see [4].

Theorem 1 (A. N. Kolmogoroff) Let $Q(x, t),(x, t) \in D$ be a random field with real or complex values and $D$ is a compact domain in $\mathbb{R}^{2}$. Assume that there exist positive constants $\gamma, C, \alpha_{1}$ and $\alpha_{2}$ with $\alpha_{1}^{-1}+\alpha_{2}^{-1}<1$ satisfying

$$
E\left|Q\left(x_{1}, t_{1}\right)-Q\left(x_{2}, t_{2}\right)\right|^{\gamma} \leq C\left[\left(x_{1}-x_{2}\right)^{\alpha_{1}}+\left(t_{1}-t_{2}\right)^{\alpha_{2}}\right]
$$

for every $\left(x_{i}, t_{i}\right) \in D$. Then $Q[x, t)$ has a continuous modification.

Let $\beta_{1}$ and $\beta_{2}$ be arbitrary positive numbers less than $\alpha_{1} c_{0}$ or $\alpha_{2} c_{0}$ respectively, $c_{0} \equiv$ $\left(1-\alpha_{1}^{-1}-\alpha_{2}^{-1}\right) / \gamma$. Then there exists a positive random variable $K$ with $E K^{\gamma}<\infty$ such that

$$
\left|Q\left(x_{1}, t_{1}\right)-Q\left(x_{2}, t_{2}\right)\right| \leq K\left[\left(x_{1}-x_{2}\right)^{\beta_{1}}+\left(t_{1}-t_{2}\right)^{\beta_{2}}\right] .
$$

Consider the wave equation $Q_{t t}-Q_{x x}=O$ with Gaussian initial data such that $Q$ is $e^{-\int Q_{x}^{2} / 2} d^{\infty} Q$, and $P$ is $e^{-\int P^{2} / 2} d^{\infty} P$ restricted to the submanifold $\hat{Q}(0)=\hat{P}(0)=0$.

The Fourier coefficients are independent complex isotropic Gaussian variables such that $\overline{\hat{Q}(n)}=\hat{Q}(-n), \overline{\hat{P}(n)}=\hat{P}(-n)$ and $E|\hat{Q}(n)| 2=n^{-2}, E|\hat{Q}(n)|^{2}=1$. Using rotation invariance of the measure and its invariance under the flow

$$
\begin{aligned}
E\left|Q\left(x, t_{1}\right)-Q\left(x, t_{2}\right)\right|^{2} & =E|Q(0, h)-Q(0,0)|^{2} \quad\left(\text { where } h=t_{2}-t_{1}\right) \\
& =E\left|\sum_{k \neq 0}(\cos k h-1) \hat{Q}(k)\right|+E\left|\sum_{k \neq 0} \frac{\sin k h}{k} \hat{P}(k)\right|^{2} \\
& =2 \sum_{k>0} \frac{(\cos k h-1)^{2}}{k^{2}}+2 \sum_{k>0} \frac{\sin ^{2} k h}{k^{2}} .
\end{aligned}
$$

The first term can be overestimated as

$$
\leq c_{1} \sum_{0<k \leq h^{-1}} \frac{(k h)^{4}}{k^{2}}+c_{2} \sum_{h^{-1}<k} \frac{1}{k^{2}} \leq c_{3} h^{4} h^{-3}+c_{4} h \leq c_{5} h .
$$

The same estimate holds for the second term. Using the Gaussian character of the field ${ }^{2}$ $Q_{W}(x, t)$ :

$$
E\left|Q_{W}\left(x, t_{1}\right)-Q_{W}\left(x, t_{2}\right)\right|^{2 n} \leq c_{n}\left|t_{1}-t_{2}\right|^{n} .
$$

Likewise

$$
E\left|Q_{W}\left(x_{1}, t\right)-Q_{W}\left(x_{2}, t\right)\right|^{2 n} \leq c_{n}\left|t_{1}-t_{2}\right|^{n} .
$$

Now apply Kolmogoroff's criteria and pass to the limit with $n \rightarrow \infty$.

$$
{ }^{2} E x^{2 n}=\frac{(2 n) !}{2^{n} n !}\left(E x^{2}\right)^{n} \text { if } x \text { is a Gaussian variable with zero mean. }
$$




\section{Step 2}

Optimality of the Hölder exponent $\frac{1}{2}$ for the space increment is a classical result. We present an elementary proof of this fact which works in other cases as well.

Note that $E|Q(\bullet, t)|_{s}^{2}=\sum E \mid \widehat{Q}(n, t)^{2}\left(1+n^{2}\right)^{s}<\infty$ if and only if $s<\frac{1}{2}$. The following fact $^{3}$ implies the rest. Let $Q(x), x \in[0,2 \pi]$ be a rotationally-invariant Gaussian process such that

$$
\left|Q\left(x_{1}\right)-Q\left(x_{2}\right)\right|<K\left(x_{1}-x_{2}\right)^{\beta}
$$

with some $K, E K^{2}<\infty$. Then $E|Q|_{s}^{2}<\infty$, for all $s<\beta$.

From the assumptions made, we get:

$$
\begin{gathered}
E \int_{0}^{2 \pi}|Q(x+h)-Q(x)|^{2} d x \leq E K^{2} h^{2 \beta}, \\
Q\left(x+\frac{\pi h}{4}\right)-Q\left(x-\frac{\pi h}{4}\right)=2 i \sum_{n \neq 0} \widehat{Q}(n) e^{i n x} \sin \frac{\pi h n}{4} .
\end{gathered}
$$

Parsevall's identity implies

$$
E \int_{0}^{2 \pi}\left|Q\left(x+\frac{\pi h}{4}\right)-Q\left(x-\frac{\pi h}{4}\right)\right|^{2} d x=4 \sum_{n \neq 0} E|\widehat{Q}(n)|^{2} \sin ^{2} \frac{\pi h n}{4} .
$$

Therefore

$$
\sum_{n \neq 0} E|\widehat{Q}(n)|^{2} \sin ^{2} \frac{\pi h n}{4} \leq c_{1} h^{2 \beta}
$$

and

$$
\sum_{\frac{1}{h} \leq n<\frac{2}{h}} E|\widehat{Q}(n)|^{2} \leq c_{2} h^{2 \beta}
$$

The substitution $h \rightarrow h / 2^{r}$ yields

$$
\sum_{\frac{2^{r}}{h} \leq n<\frac{2^{r}+1}{h}} E|\widehat{Q}(n)|^{2} \leq c_{3} \frac{h^{2 \beta}}{4^{\beta r}} .
$$

Finally

$$
\sum_{\frac{1}{h} \leq n} E|\widehat{Q}(n)|^{2} \leq \sum_{r=0}^{\infty} \sum_{\frac{2^{r}}{h} \leq n<\frac{2^{r+1}}{h}} E|\widehat{Q}(n)|^{2} \leq c_{4} h^{2 \beta}
$$

and $^{4}$

$$
\sum_{k \leq|n|} E|\hat{Q}(n)|^{2} \leq c_{4} \frac{1}{k^{2 \beta}}
$$

${ }^{3}$ This is stochastic version of the classical embeding theorem, [7].

${ }^{4}$ In the proof of this estimate we borrowed the idea from [1, section 82$]$. 
It implies for positive $n$ :

$$
S(n) \equiv \sum_{n \leq k}|\hat{Q}(k)|^{2} \leq c_{4} \frac{1}{n^{2 \beta}} .
$$

By Abel's summation formula for positive $M$ and $N$, we have

$$
\begin{aligned}
\sum_{M}^{N} E|\hat{Q}(n)|^{2}\left(1+n^{2}\right)^{s}= & S(M)\left(1+M^{2}\right)^{s}-S(N+1)\left(1+N^{2}\right)^{s} \\
& +\sum_{M+1}^{N} S(n)\left[\left(1+n^{2}\right)^{s}-\left(1+(n-1)^{2}\right)^{s}\right] \\
\leq & S(M)\left(1+M^{2}\right)^{s}+\sum_{M+1}^{N} S(n)\left[\left(1+n^{2}\right)^{s}-\left(1+(n-1)^{2}\right)^{s}\right] .
\end{aligned}
$$

For big $n$

$$
\left(1+n^{2}\right)^{s}-\left(1+(n-1)^{2}\right)^{s}=\left(1+n^{2}\right)^{s}\left[\frac{2 s}{n}+O\left(\frac{1}{n^{2}}\right)\right] .
$$

This together with the estimate for $S(n)$ implies

$$
\sum_{M}^{+\infty} E|\widehat{Q}(n)|^{2}\left(1+n^{2}\right)^{s}<\infty, \text { for } s<\beta .
$$

Negative indexes are handled in the same way. The proof is finished.

For any fixed $x, Q_{W}(x, \bullet)$ is a $2 \pi$-periodic rotationally invariant Gaussian process such that $E\left|\hat{Q}_{W}(x, n)\right|^{2}=n^{-2}$. The same arguments used above show optimality of the exponent in the time increment.

\section{Step 3}

First, we estimate Hölder exponents for a solution of the nonlinear equation. Let $h=$ $t_{1}-t_{2}$, using invariance of the measure

$$
\begin{aligned}
E\left|Q\left(x_{1}, t\right)-Q\left(x_{2}, t\right)\right|^{2 n}= & E\left|Q_{0}\left(x_{1}\right)-Q_{0}\left(x_{2}\right)\right|^{2 n} \leq c_{n}\left(x_{1}-x_{2}\right)^{n} \\
E\left|Q\left(x, t_{1}\right)-Q\left(x, t_{2}\right)\right|^{2 n}= & E\left|Q(x, h)-Q_{0}(x)\right|^{2 n} \\
\leq & c_{n} E\left|\sin \frac{\sqrt{-\partial_{x}^{2}} h}{\sqrt{-\partial_{x}^{2}}} P_{0}(x)+\cos \sqrt{-\partial_{x}^{2}} h Q_{0}(x)-Q_{0}(x)\right|^{2 n} \\
& +c_{n} E\left|\int_{0}^{h} d s \frac{1}{2} \int_{x-(h-s)}^{x+(h-s)} d y f(Q(y, s))\right|^{2 n} .
\end{aligned}
$$

To estimate the first term replace the measure $e^{-\int F} \times e^{-\int Q_{x}^{2} / 2} d^{\infty} Q$ by $C e^{-k \int Q^{2}} \times$ $e^{-\int Q_{x}^{2} / 2} d^{\infty} Q$ with some big $C$ and proceed like in Step 1. To estimate the second term use Hölder's inequality and $E|f(Q)|^{2 n}<\infty$ for every $n$. Eventually

$$
E\left|Q\left(x, t_{1}\right)-Q\left(x, t_{2}\right)\right|^{2 n} \leq c_{n}\left(t_{1}-t_{2}\right)^{n} .
$$


Kolmogoroff's criteria implies that $Q(x, t)$ satisfies (1) with the same Hölder exponents not exceeding $1 / 2$. The last statement concerning derivatives $\partial_{x} N(x, t)-\partial_{t} N(x, t)$ follows from the explicit formulas

$$
\begin{aligned}
& \partial_{x} N(x, t)=-\frac{1}{2} \int_{0}^{h} d s[f(Q(x+(h-s), s))-f(Q(x-(h-s), s))], \\
& \partial_{t} N(x, t)=-\frac{1}{2} \int_{0}^{h} d s[f(Q(x+(h-s), s))+f(Q(x-(h-s), s))],
\end{aligned}
$$

and locally Lipschitz character of $f$. The proof is completed.

\section{Nonlinear Schrödinger equations}

The next point if the discussion is 1D nonlinear Schrödinger equation

$$
i \psi_{t}=-\psi_{x x}+f\left(|\psi|^{2}\right) \psi
$$

where $\psi(x, t)$ is a complex function $\psi=Q+i P$ which satisfies periodic boundary conditions $\psi(0, t)=\psi(2 \pi, t)$. It can be written in the Hamiltonian form

$$
\psi_{t}=\{\psi, H\}
$$

with the Hamiltonian $H=\frac{1}{2} \int_{0}^{2 \pi}\left|\psi_{x}\right|^{2}+F\left(|\psi|^{2}\right) d x, F^{\prime}=f$ and a bracket

$$
\{A, B\}=2 i \int_{0}^{2 \pi}\left[\frac{\partial A}{\overline{\psi(x)}} \frac{\partial B}{\psi(x)}-\frac{\partial A}{\psi(x)} \frac{\partial B}{\bar{\psi}(x)}\right] d x .
$$

An invariant Gibbs' state $e^{-H} d^{\infty} \psi d^{\infty} \bar{\psi}$ was constructed in [3, 5] under the assumption that $F \geq 0$ is an even polynomial. The Gibbs' state is a product of two independent circular Brownian motions on $Q$ and $P$ whose components are coupled together by the nonlinear factor $e^{-\int F\left(Q^{2}+P^{2}\right)}$.

Written in the integral form the equation is

$$
\begin{aligned}
\psi(x, t) & =e^{i \partial_{x}^{2} t} \psi_{0}(x)-i \int_{0}^{t} e^{i \partial_{x}^{2}(t-s)} f\left(|\psi|^{2}\right) \psi(x, s) d s \\
& =\psi_{S}(x, t)+N(x, t),
\end{aligned}
$$

where $\psi_{0}(x)$ is initial data. The solution of the free Schrödinger equation satisfies

$$
\left|\psi_{S}\left(x_{1}, t_{1}\right)-\psi_{S}\left(x_{2}, t_{2}\right)\right| \leq K\left(\left|x_{1}-x_{2}\right|^{\beta_{1}}+\left|t_{1}-t_{2}\right|^{\beta_{2}}\right)
$$

with $0<\beta_{1}<\frac{1}{2}, 0<\beta_{2}<\frac{1}{4}$, and random constant $K, E K^{2}<\infty$, which depend on $\beta$ 's. The exponents $\frac{1}{2}, \frac{1}{4}$ are optimal. The same can be said about $\psi(x, t)$, a solution of NLS itself. The proof of this statements is similar to the corresponding one for the nonlinear wave equation.

The nonlinear term $N(x, t)$ seems to be smoother then $\psi_{S}(x, t)$. This implies that the microstructure of the field $\psi(x, t)$ is determined by the linear term $\psi_{S}(x, t)$, but the proof is not known. Presumably, Hölder exponents for $N(x, t)$ depend on arithmetical properties of the coefficients of the polynomial $F$. There is no uniform smoothing as one can see from the following example. 


\section{Example}

Let $\Gamma(x, t) \equiv \sum_{n \neq 0} e^{i n x} e^{-i\left(n^{2}+n^{\alpha}\right) t} \widehat{\psi}_{0}(n)$, arbitrary $\alpha \geq 0$ and $\widehat{\psi}_{0}(n)$ are independent complex isotropic Gaussian variables, $E\left|\widehat{\psi}_{0}(n)\right|^{2}=\frac{1}{1+n^{2}}$. The Gaussian field $\Gamma(x, t), x \in$ $[0,2 \pi], s \in \mathbb{R}^{1}$ is stationary in time and rotationally invariant; $\Gamma(\bullet, t)$ is a complex OrnsteinUhlenbeck process with zero mean for any $t$. By straightforward computation

$$
\begin{aligned}
N(x, t) & =-i \int_{0}^{t} e^{i \partial_{x}^{2}(t-s)} \Gamma(x, s) d s \\
& =-i \int_{0}^{t} \sum_{n \neq 0} e^{i n x} e^{-i n^{2}(t-s)} e^{-i\left(n^{2}+n^{\alpha}\right) s} \widehat{\psi}_{0}(n) d s \\
& =-i \sum_{n \neq 0} e^{i n x} e^{-i n^{2} t} \widehat{\psi}_{0}(n) \frac{e^{-i n^{\alpha} t}-1}{-i n^{\alpha}} .
\end{aligned}
$$

We see that $N(\bullet, t)$ gains $\alpha$ Sobolev's exponents in comparison with $\Gamma(\bullet, t)$.

\subsection{KdV-type equations}

The last topic of the discussion to KdV-type equations

$$
Q_{t}=-Q_{x x x}+(f(Q))_{x}
$$

with periodic boundary conditions $Q(0, t)=Q(2 \pi, t)$. The equation can be written in the Hamiltonian form

$$
Q_{t}=\{Q, H\}
$$

with the Hamiltonian $H=\int_{0}^{2 \pi} \frac{Q_{x}^{2}}{2}+F(Q) d x, F^{\prime}=f$ and a bracket

$$
\{A, B\}=\int_{0}^{2 \pi} \frac{\partial A}{\partial Q(x)} \partial_{x} \frac{\partial A}{\partial Q(x)} d x
$$

An invariant Gibbs state $e^{-H} d^{\infty} Q$ was constructed in [3] for particular nonlinearities $F(Q)=Q^{3} / 3(\mathrm{KdV})$ and $F(Q)=Q^{4} / 4$ (modified $\left.\mathrm{KdV}\right)$. The measure is a circular Brownian motion $e^{-\int Q_{x}^{2} / 2} d^{\infty} Q$ multiplied by the nonlinear term $e^{-\int F(Q)}$.

The equation can be written in the integral form

$$
\begin{aligned}
Q(x, t) & =e^{-\partial_{x}^{3} t} Q_{0}(x)+\partial_{x} \int_{0}^{t} e^{-\partial_{x}^{3}(t-s)} f(Q(x, s)) d s \\
& =Q_{A}(x, t)+U[f](x, t)
\end{aligned}
$$

According to J. Bourgain (private communication) the solution $Q(x, t)$ will be continuous in space-time. The solution of the linear Airy equation satisfies

$$
\left|Q_{A}\left(x_{1}, t_{1}\right)-Q_{A}\left(x_{2}, t_{2}\right)\right| \leq\left(\left|x_{1}-x_{2}\right|^{\beta_{1}}+\left|t_{1}-t_{2}\right|^{\beta_{2}}\right)
$$


with the optimal bounds $0<\beta_{<} \frac{1}{2}, 0<\beta_{2}<\frac{1}{6}$, and some random constant $K, E K^{2}<\infty$, which depend on $\beta$ 's. Nothing is known about smoothness of the nonlinear term $U[f](x, t)$. To get some idea consider the KdV equation. In symbolic form

$$
\begin{aligned}
Q & =Q_{A}+U\left[Q^{2}\right] \\
& =Q_{A}+U\left[\left(Q_{A}+U\left[Q^{2}\right]\right)^{2}\right] \\
& =Q_{A}+U\left[Q_{A}^{2}\right]+U\left[2 Q_{A} U\left[Q^{2}\right]+U^{2}\left[Q^{2}\right]\right] \\
& =Q_{A}+U\left[Q_{A}^{2}\right]+\ldots
\end{aligned}
$$

Now look at $U\left[Q_{A}^{2}\right]$, the first term in the "approximation". We will prove

$$
\begin{aligned}
& E\left(U\left[Q_{A}^{2}\right]\left(x_{1}, t\right)-U\left[Q_{A}^{2}\right]\left(x_{2}, t\right)\right)^{2} \leq C\left|x_{1}-x_{2}\right|, \\
& E\left(U\left[Q_{A}^{2}\right]\left(x_{1}, t\right)-U\left[Q_{A}^{2}\right]\left(x_{2}, t\right)\right)^{4} \leq C(\epsilon)\left|x_{1}-x_{2}\right|^{(2-\epsilon)},
\end{aligned}
$$

for any $\epsilon>0$. This indicates that $U\left[Q_{A}^{2}\right](\bullet, t)$ is $x$-continuous due to (5) by Kolmogoroff and similar to the Brownian motion because of (4). It is possible that in this case the local structure of the field $Q(x, t)$ depends on the nonlinear term $N(x, t)$.

To prove (4) and (5) we need Wick's theorem, see [2].

Theorem 2 (Wick) Let $\xi_{1}, \xi_{2}, \ldots, \xi_{2 n}$ are real or complex Gaussian variables with zero mean, then

$$
E \xi_{1} \times \cdots \times \xi_{2 n}=\frac{1}{2^{n} n !} \sum_{\mu} E \xi_{\mu_{1}} \xi_{\mu_{2}} \times \cdots \times E \xi_{\mu_{2 n-1}} \xi_{\mu_{2 n}},
$$

where summation is taken over the permutation group of $2 n$ elements.

Let $Q_{A}(x, t)=\sum_{n \neq 0} e^{i n x} e^{i n^{3} t} \widehat{Q}_{0}(n)$ where $\widehat{Q}_{0}(n)$ is a Gaussian complex isotropic variable, $\widehat{Q}_{0}(n)=\hat{Q}_{0}(-n), E\left|\widehat{Q}_{0}(n)\right|^{2}=\frac{1}{1+n^{2}}$. Then

$$
\begin{aligned}
U\left[Q_{A}^{2}\right](x, t) & =\partial_{x} \int_{0}^{t} e^{-\partial_{x}^{3}(t-s)} Q_{A}^{2}(x, s) d s \\
& =\sum_{n \neq 0} e^{i n x} \sum_{\substack{n_{1}+n_{2}=n \\
n_{i} \neq 0}} \hat{Q}_{0}\left(n_{1}\right) \hat{Q}_{0}\left(n_{2}\right) \frac{e^{i\left(n_{1}^{3}+n_{2}^{3}\right) t}-e^{i n^{3} t}}{n_{1}^{3}+n_{2}^{3}-n^{3}} .
\end{aligned}
$$

Using the arithmetical fact $n_{1}^{3}+n_{2}^{3}-n^{3}=-3 n n_{1} n_{2}$ we obtain

$$
U\left[Q_{A}^{2}\right](x, t)=\sum_{n \neq 0} e^{i n x} \sum_{\substack{n_{1}+n_{2}=n \\ n_{i} \neq 0}} \hat{Q}_{0}\left(n_{1}\right) \hat{Q}_{0}\left(n_{2}\right) M\left(n_{1}, n_{2}, t\right),
$$

where

$$
M\left(n_{1}, n_{2}, t\right)=\frac{e^{i\left(n_{1}^{3}+n_{2}^{3}\right) t}-e^{i n^{3} t}}{-3 n_{1} n_{2}} .
$$

Note $\left|M\left(n_{1}, n_{2}, t\right)\right| \leq 2$ if $n_{1} n_{2} \neq 0$. 
First, we prove that $E\left(U\left[Q_{A}^{2}\right](x, t)\right)^{2}$ is finite. Using rotational invariance of the measure

$$
\begin{aligned}
E\left(U\left[Q_{A}^{2}\right](x, t)\right)^{2} & =E\left(U\left[Q_{A}^{2}\right](0, t)\right)^{2} \\
& =\sum_{n_{1}, n_{2} \neq 0} \sum_{\begin{array}{c}
p_{1}+p_{2}=n_{1} \\
p_{3}+p_{4}=n_{2} \\
p_{i} \neq 0
\end{array}} E \hat{Q}_{0}\left(p_{1}\right) \hat{Q}_{0}\left(p_{2}\right) \hat{Q}_{0}\left(p_{3}\right) \hat{Q}_{0}\left(p_{4}\right) M\left(p_{1}, p_{2}\right) M\left(p_{3}, p_{4}\right) .
\end{aligned}
$$

By Wick's rule

$$
E \hat{Q}_{0}\left(p_{1}\right) \hat{Q}_{0}\left(p_{2}\right) \hat{Q}_{0}\left(p_{3}\right) \hat{Q}_{0}\left(p_{4}\right)=\frac{1}{2^{2} 2 !} \sum_{\mu} E \hat{Q}_{0}\left(p_{\mu_{1}}\right) \hat{Q}_{0}\left(p_{\mu_{2}}\right) \times E \hat{Q}_{0}\left(p_{\mu_{3}}\right) \hat{Q}_{0}\left(p_{\mu_{4}}\right) .
$$

The sum venishes unless $n_{1}=-n_{2}$ and $p_{1}=-p_{3}, p_{2}=-p_{4}$ or $p_{1}=-p_{4}, p_{2}=-p_{3}$. Therefore

$$
\begin{aligned}
& E \hat{Q}_{0}\left(p_{1}\right) \hat{Q}_{0}\left(p_{2}\right) \hat{Q}_{0}\left(p_{3}\right) \hat{Q}_{0}\left(p_{4}\right) \\
& \quad=E\left|\hat{Q}_{0}\left(p_{1}\right)\right|^{2} E\left|\hat{Q}_{0}\left(p_{2}\right)\right|^{2}=\left\{\begin{array}{cl}
\frac{1}{1+p_{1}^{2}} \frac{1}{1+p_{2}^{2}}, & \text { if } p_{1} \neq p_{2} \\
2 \frac{1}{1+p_{1}^{2}} \frac{1}{1+p_{2}^{2}}, & \text { if } p_{1}=p_{2}
\end{array}\right.
\end{aligned}
$$

and

$$
\begin{aligned}
& E\left(U\left[Q_{A}^{2}\right](x, t)\right)^{2} \leq 2 \sum_{n \neq 0} \sum_{\substack{p_{1}+p_{2}=n \\
p_{i} \neq 0}} \frac{1}{1+p_{1}^{2}} \frac{1}{1+p_{2}^{2}}\left|M\left(p_{1}, p_{2}\right)\right|^{2} \\
& \leq 8 \sum_{n \neq 0} \sum_{\substack{p_{1}+p_{2}=n \\
p_{i} \neq 0}} \frac{1}{1+p_{1}^{2}} \frac{1}{1+p_{2}^{2}} \leq c_{1} \sum_{n \neq 0} \frac{1}{n^{2}}<\infty .
\end{aligned}
$$

In the last estimate we used

$$
\int_{-\infty}^{+\infty} \frac{1}{1+x^{2}} \frac{1}{1+(n-x)^{2}} d x=\frac{2 \pi}{n^{2}+4}
$$

The estimate for the second moment of the increment is similar.

$$
\begin{aligned}
& E\left(U\left[Q_{A}^{2}\right](h, t)-U\left[Q_{A}^{2}\right](0, t)\right)^{2}=\sum_{n_{1}, n_{2} \neq 0}\left(e^{i n_{1} h}-1\right)\left(e^{i n_{2} h}-1\right) \times \\
& \times \sum_{\substack{p_{1}+p_{2}=n_{1} \\
p_{3}+p_{4}=n_{2} \\
p_{1} \neq 0}} E \hat{Q}_{0}\left(p_{1}\right) \hat{Q}_{0}\left(p_{2}\right) \hat{Q}_{0}\left(p_{3}\right) \hat{Q}_{0}\left(p_{4}\right) M\left(p_{1}, p_{2}\right) M\left(p_{3}, p_{4}\right) \\
& \leq 2 \sum_{n \neq 0}\left(e^{i n h}-1\right)\left(-e^{i n h}-1\right) \sum_{\substack{p_{1}+p_{2}=n \\
p_{i} \neq 0}} \frac{1}{1+p_{1}^{2}} \frac{1}{1+p_{2}^{2}}\left|M\left(p_{1}, p_{2}\right)\right|^{2} .
\end{aligned}
$$

Finally

$$
E\left(U\left[Q_{A}^{2}\right](h, t)-U\left[Q_{A}^{2}\right](0, t)\right)^{2} \leq c_{2} \sum_{|n|<h^{-1}} \frac{\left|e^{i h n}-1\right|}{n^{2}}+c_{3} \sum_{h^{-1} \leq|n|} \frac{1}{n^{2}} \leq c_{4} h .
$$

The proof of (5) can be obtained by the same methods.azw 


\section{References}

[1] Akhiezer, N.I. (1956) Lectures on Approximation Theory. Frederic Ungar Publishing Co., New York

[2] Bessis, D.; Itzykson, C.; Zuber, J.B. (1980) "Quantum field theory in graphical enumeration", Advances in Applied Mathematics 1: 109-157.

[3] Bourgain, J. (1994) "Periodic nonlinear Schrödinger equation and invariant measures", Comm. Math. Phys. 166: 1-26.

[4] Kunita, H. (1990) Stochastic Flows and Stochastic Differential Equations. Cambridge University Press, Cambridge.

[5] McKean, H.P. (1994) "Statistical mechanics of nonlinear wave equations (4): cubic Schrödinger", Comm. Math. Phys. 220: 2-13.

[6] McKean, H.P.; Vaninsky, K.L. (1994) "Statistical mechanics of nonlinear wave equation (1)", in: L. Sirovich (Ed.) Papers Dedicated to F. John. Trends and Perspectives in Applied Mathematics, Springer-Verlag, New York: 239-264.

[7] Stein, E.M. (1970) Singular Integrals and Differentiability Properties of Functions. Princeton University Press, Princeton NJ. 